\title{
Stochastic Look-ahead Scheduling Method for Linear Construction Projects
}

\author{
Lingguang Song* and Sang-Hoon Lee \\ Department of Construction Management, University of Houston, 375 T2 \\ Houston, TX 77204, USA \\ *E-mail: lsong5@uh.edu \\ Fitria H. Rachmat \\ Bechtel Corporation \\ Houston, TX 77056, USA \\ E-mail: fhrachma@bechtel.com \\ Received 16 March 2012 \\ Accepted 16 September 2012
}

\begin{abstract}
Many construction projects involve repetitive operations that are exposed to various constraints and uncertainties. During construction field execution, schedulers must maintain and update look-ahead schedules, which coordinate work for the next several weeks based on the most recent productivity achieved. This study enhances the traditional linear scheduling method used for scheduling repetitive construction operations with stochastic simulation to incorporate uncertainty in activity performance during field operations. The proposed Stochastic Linear Scheduling Method (SLSM) was implemented and demonstrated in a pipeline construction project.
\end{abstract}

Keywords: linear construction projects, linear scheduling, simulation, pipeline construction.

\section{Introduction}

Linear construction projects refer to construction work that involves a number of correlated activities performed in succession and repeated throughout the entire project duration ${ }^{1}$, such as the construction of high-rise buildings, tunnels, roadways, and pipe/sewer lines. Due to technical, resource, and space constrains, these repetitive activities are typically inter-dependent, i.e. delay or slow progress of one activity will affect its successors' performance, and eventually impact the overall project duration. An effective coordination of various repetitive activities and their interactions through careful planning and scheduling is critical to project success. Poor scheduling can result in considerable waste as laborers and equipment become idle due to delayed completion of preceding tasks, limited availability of needed resources, or other space and time constraints.
Two types of schedules are frequently used in a construction project: master schedules and look-ahead schedules $^{1}$. A project master schedule provides management with the big picture of project time and procedure. A look-ahead schedule, or short-interval schedule, a schedule maintained during the actual construction operation, is an extension from the master schedule that specifies the day-to-day details of work scopes, resource allocation, and project team coordination for the upcoming several weeks or months. A look-ahead schedule should be developed based on the most recent project productivity data, and it must be updated periodically so that it can accurately reflect the current project status and provide a reliable forecast for future project performance. Compared with a master schedule, a look-ahead schedule provides the operationlevel managers a more detailed and realistic view of future performance and potential problems so that waste and delays can be identified and eliminated. Therefore, 
effective look-ahead scheduling based on the most recent performance data can thus help to ensure a smooth and continuous work flow.

Traditionally, look-ahead scheduling has been presented in a bar chart or Critical Path Method (CPM) formats. However, the Linear Scheduling Method (LSM) provides an alternative approach ${ }^{1}$. LSM is a graphical tool designed specifically for scheduling repetitive linear construction projects, such as pipeline projects. In this research, LSM is used as the underlying algorithm for look-ahead scheduling. Pipeline construction projects and LSM are further described in the next section.

Regardless of the schedule format, the current industry practice of look-ahead scheduling is based on a deterministic analysis of actual project data-i.e., forecasting future performance using average past performance. This deterministic method is unable to consider the variability of productivity in forecasting future performance, which may result in a considerable amount of work interruption and crew idle time. This study enhances the traditional LSM with stochastic simulation to incorporate uncertainty in look-ahead scheduling. Further, this approach is demonstrated in an actual pipeline construction project case study. An introduction to pipeline construction, LSM, and past relevant studies is presented in the next section. This is followed by a description of the proposed stochastic linear scheduling method and the case study.

\section{Background}

\subsection{Pipeline Construction}

Pipeline construction is a highly repetitive and resourceintensive process that is exposed to various constraints and uncertainties in the working environment. A pipeline construction project operates like a moving assembly line, involving a variety of sequenced tasks and specialized crews, as shown in Figure 1 . The specific location of the pipeline route is first identified and marked with stakes. The pipeline right-of-way is cleared of vegetation and graded to ensure a leveled base. Backhoes or trenching machines are then used to excavate a pipeline trench. This is followed by stringing the individual pipe sections along the trench line. Individual joints of pipe may also be bent to a desired angle, if required. After stringing and bending are completed, the pipe sections are welded together, coated, inspected, and placed on temporary supports along the edge of the trench. The pipe assembly is then lowered into the trench and tied into the preceding pipe section. The trench is then backfilled. After backfilling and cleaning up, the pipeline is hydrostatically tested for strength and leaks.

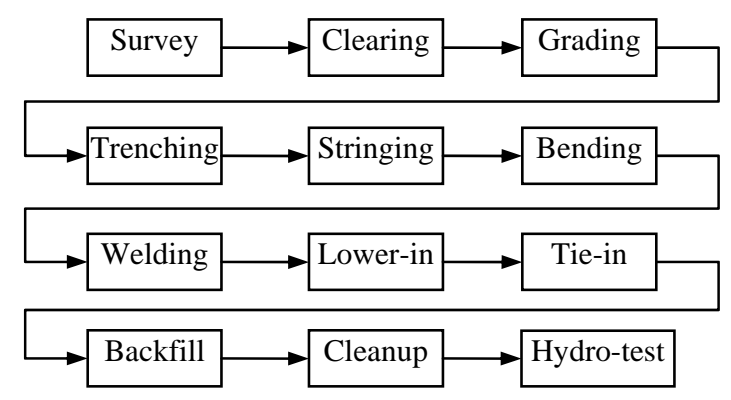

Fig. 1. Pipeline construction process.

\subsection{Linear Scheduling Method (LSM)}

LSM is designed for scheduling linear construction projects that contain a family of repetitive and nearly identical tasks ${ }^{1}$. It represents a repetitive activity as a production line in a two-dimensional time and space graph, as illustrated in Figure 2. The horizontal axis represents time, and the vertical axis is the location of an activity or a crew. The slope of a production line represents its productivity rate. A production line may be a straight line if the productivity rate is constant. However, in real-world operations, a production line typically features varying slopes due to the variability in productivity rates, which can be attributed to many factors, such as quantity and complexity of work and weather conditions. The horizontal distance between two lines is a graphic representation of the time float, or time buffer, between the activities. Similarly, the vertical distance represents the physical distance, or space buffer, between the activities. Much previous research has shown that LSM allows better representation and visualization of scheduling information than the conventional CPM or bar charts in terms of time and space constraints, activity location, and productivity rates ${ }^{2}$. It is important that work continuity be maintained in order to achieve efficiency in a linear construction project. In this regard, LSM is particularly useful in visualizing work flow and time and space constraints graphically so that a scheduler can easily adjust activity start time or balance productivity rates to achieve work continuity. Therefore, LSM is 
used in this research for look-ahead scheduling of linear construction projects, e.g. pipeline construction presented in the case study.

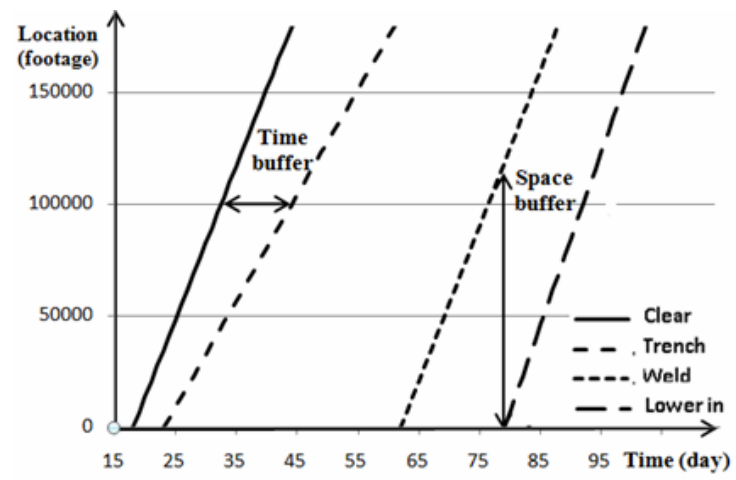

Fig. 2. A sample LSM chart.

\subsection{Related Research}

Though look-ahead scheduling has been used for many years in construction, few publications provide information on it. In fact, the few sources that addressed the topic did little more than provide definitions ${ }^{1}$. Schedulers typically rely on readily available project progress data and their own subjective judgments in forecasting future performance, and the resulting lookahead schedule is deterministic in nature.

Past research in LSM has been focused on applying LSM in early project planning, but uncertainty that is frequently encountered during field operations has not been fully addressed. Srisuwanrat and Ioannou ${ }^{3}$ studied the optimization of repetitive schedules when activity durations are probabilistic. In this study, only precedence logic relationships between activities were modeled, and productivity rates per day were assumed to follow the normal distribution only, which may not represent real-world cases. Similarly, Liu et al. ${ }^{4}$ also studied the linear schedule optimization problem. To calculate the production rate, a chaotic function is used to simulate the uncertainties and the correlations in a time series of points. However, for look-ahead scheduling, the most accurate data can be obtained from real-world operations and they should be used to model and forecast production variations. Duffy et al. ${ }^{5}$ studied LSM with varying production rates in regards to when and where they occur in time and space for a given linear construction project. The changes of productivity rate is either subjectively defined or through regression analysis based on historical data. While complementary to the effort in our study, their work did not explicitly model the uncertainty in productivity changes and, hence, the output from the method is still a point estimate of future performance. El-Sayegh ${ }^{6}$ developed deterministic and probabilistic models for calculating resource-based linear schedules. The deterministic model can be used to produce a linear schedule based solely on user input. The probabilistic model may be used to produce a linear schedule based on Monte Carlo simulation, which accounts for variability and uncertainty of construction projects. To make LSM suitable for practical look-ahead scheduling of horizontal linear construction projects (e.g., pipeline construction), our research here enhances the previous research in two areas: (1) formalizing the procedure of productivity data collection and analysis for measuring productivity variability in repetitive activities, and (2) modeling both time and space buffer constraints between activities while considering productivity variability. The following two sections describe the proposed stochastic linear scheduling procedure along with a case study applying the method to pipeline construction.

\section{Stochastic Linear Scheduling Method}

\subsection{System Procedure}

The proposed Stochastic Linear Scheduling Method (SLSM) enhances the traditional LSM with the capability of modeling the variability in activity performance based on actual productivity data. Simulation input modeling provides a way for a scheduler to measure and evaluate the variability in activity performance and later to incorporate it into look-ahead scheduling. To achieve this, actual project performance data must be properly collected and analyzed in order to measure the variability. Once productivity data are collected, they will be analyzed through a standard simulation input modeling procedure, which involves fitting the productivity data to standard statistical distributions and then determining the bestfitted distribution based on goodness-of-fit tests. The information received from input modeling allows schedulers to represent the variability of activity performance in the form of probability distributions. Interested readers can refer to Law and Kelton ${ }^{7}$ for more technical details about the aforementioned simulation input modeling procedure. The data collection and input 
modeling procedures are described and demonstrated through the case study later in this paper.

For look-ahead scheduling purposes, the impact of the variability of activity productivity rates on future project performance is further evaluated through discrete-event simulation in SLSM. Discrete-event simulation is a mathematical-logical model representing a real-world system that evolves over time, allowing users to experiment with the model to analyze and predict system performance ${ }^{4}$. To facilitate the use of SLSM, a computer program, Stochastic Linear Scheduling Method (SLSM), was designed and implemented in this study. The program allows a scheduler to model and analyze repetitive linear construction projects using graphic modeling tools while considering variability in activity performance. This computerized tool and its development and application is described in the following section.

Once the model is established, the scheduler can conduct "what-if" analysis or risk analysis by experimenting with the computerized simulation and observing its behavior. For example, experiments can be conducted to evaluate baseline schedule, total project duration, individual activity performance, and activity idle time. Accurate look-ahead scheduling can help schedulers to better anticipate problem areas and formulate new plans to improve overall project performance.

\subsection{Simulation Program}

The SLSM program was implemented within a simulation tool development environment, Simphony, which allows a developer to design highly flexible simulation tools supporting graphical and hierarchical modeling ${ }^{8}$. The developed SLSM program can accurately model repetitive activities, precedence relationships, and time and space buffers, and it allows users to experiment with the model and observe outputs through a variety of output reports. SLSM contains two fundamental modeling elements: Project and Activity, as described in Table 1. Using these two elements, a scheduler can assemble a model to graphically represent repetitive activities involved in a linear construction project.
Table 1. SLSM modeling elements.

\begin{tabular}{ll}
\hline Element & \multicolumn{1}{c}{ Description } \\
\hline Project & $\begin{array}{l}\text { The Project element represents the linear } \\
\text { construction project and is the parent element of } \\
\text { any Activity element. Users can specify the } \\
\text { total quantity of work for the project in the } \\
\text { Project element. The element also handles } \\
\text { collecting simulation results and exporting } \\
\text { these results to an external database. }\end{array}$ \\
\hline Activity & $\begin{array}{l}\text { This is a generic element that can represent } \\
\text { either a predecessor or successor. Each instance } \\
\text { of the Activity element has an input port and an } \\
\text { output port. When the output of an Activity } \\
\text { element is connected to the input of another } \\
\text { activity element, the former Activity element } \\
\text { becomes the predecessor, while the latter } \\
\text { element becomes the successor. If the input of } \\
\text { an element does not connect to any Activity } \\
\text { element, this activity becomes the first activity } \\
\text { of the project and its daily start and finish } \\
\text { events are controlled only by the total work } \\
\text { quantity. The activation of other Activity } \\
\text { elements with predecessors is determined by } \\
\text { the performance of their predecessors and user- } \\
\text { defined time and/or space buffers. }\end{array}$ \\
\hline
\end{tabular}

When two activity elements are connected, the preceding activity becomes predecessor and the other activity is recognized as successor. As discussed in the background section on LSM, the predecessor and the successor usually cannot overlap, and proper space, time buffers, or both must be maintained so that the successor can be performed efficiently and safely. The time and space buffers are user-defined based on past project experience. Once determined, schedulers can specify these buffer values and associated them with the successor activity.

The simulation procedure of this predecessorsuccessor interaction through time and space buffers is illustrated in Figure 3. The time unit used in this simulation is one day, i.e. the simulation resolution is one day. This means the simulation clock advanced one day at a time. The simulation starts on Day 1 and the activity without any predecessor is recognized as the first activity in the project. Due to time and/or space buffers, we assume no other activity will start on Day 1. For the first activity during the first day, its progress during the day (e.g. footage of trenching completed) is determined by a random number (e.g. footage/day) according to the activity's input model. Then the simulation advances to the second day and a check of successor activities is conducted. If the time and/or space buffer requirements are satisfied, the successor 
can start and make a progress for one day, which is determined by their input models. This process is repeated until project completion. This completes one simulation experiment, which represents one possible occurrence in the real-world. This experiment should be repeated, e.g. 30 times or more, so that we can determine the "average" system performance.

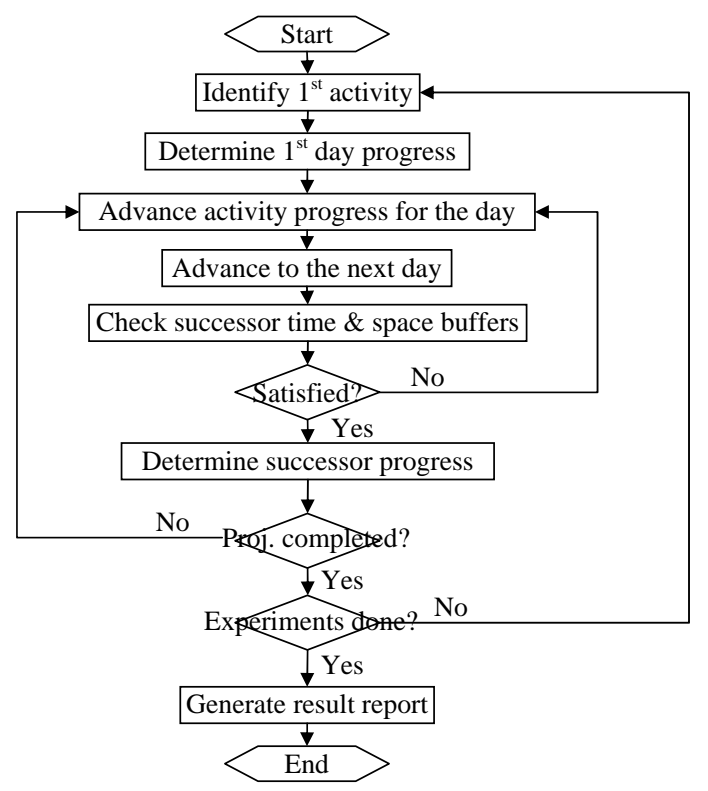

Fig. 3. Simulation procedure.

For the current version of the program, the model output data are exported to a Microsoft Access database where graphic results such as an LSM chart can be produced.

\section{Case Study of Pipeline Construction}

A pipeline construction project was selected to demonstrate and evaluate the applicability of the proposed SLSM for look-head scheduling. The sample project involves the construction of approximately 130 miles of 30-inch pipeline. The case study was focused on understanding the current practice of look-ahead scheduling, collecting actual performance data, and demonstrating the usage of SLSM.

In this project, the contractor was responsible for main pipe installation activities such as trenching, stringing, bending, pipe laying, backfilling, tie-in, cleaning, and hydro-testing. Several subcontractors were also involved in this project for activities such as horizontal directional drilling, concrete work, and coating.
The original look-ahead schedule was prepared by the contractor in a table format on a weekly basis. The look-ahead schedule contains information on upcoming activities, mile-post targets, and comments. Three sources of information are collected in preparing the look-ahead schedule. The first source of information is the most recent activity productivity data, monitored daily by the contractor and expressed in terms of footage per day. The second source of information is a list of potential operation problems identified through discussion among field managers, superintendents, and subcontractors at the weekly meeting. The third source of data is the forecast of upcoming work performance based on a scheduler's evaluation of each activity's recent productivity and its predecessor's progress. The productivity forecast is calculated deterministically as a moving average of the previous 3 to 5 weeks' productivity rates.

In a linear construction project, maintaining a crew's continuous performance is essential to achieving the activity milestones because successor activities can only be performed after their predecessors are completed. Therefore, achieving reliable milestones for each activity is extremely desirable for maintaining a smooth operation and minimizing the overall project duration. As mentioned previously, LSM is an effective scheduling method for linear construction work and, therefore, it was proposed to the contractor as an alternative look-ahead scheduling method. Furthermore, the proposed SLSM allows schedulers to evaluate the impact of the variability of project performance and take proactive measures to prevent work interruptions and thus reduce crew idle time. The application of SLSM in the sample project is described in the following sections.

\subsection{Data Collection}

In the sample project, the contractor already had a wellstructured progress-monitoring and reporting system that continuously captured performance data that could be used to model the variability of productivity rates. Physical construction progress data were collected through the Construction Daily Progress Report prepared by superintendents. In addition to progress data, this report also contained information about working conditions, quality, safety, and regulatory compliance. Daily construction progress is measured by "From" and "To" stations, which represent the start location and the end location of a crew in a working day, 
respectively. Table 2 shows a sample of daily progress data, from which the productivity rate measured by footage per day can be easily derived. Productivity rates were reported in the same way for other activities, such as clearing, grading, trenching, bending, welding, lower-in, tie-in, backfill, and cleanup. At the time of this study, about 2 months of data were collected for the above-mentioned activities as they progressed to different locations.

Table 2. Daily progress and productivity.

\begin{tabular}{|c|c|c|c|c|c|}
\hline \multirow{2}{*}{ Date } & \multirow{2}{*}{ Task } & \multicolumn{2}{|c|}{ Station } & \multirow{2}{*}{ Footage } & $\begin{array}{c}\text { Productivity } \\
(\mathrm{ft} / \mathrm{d})\end{array}$ \\
\cline { 3 - 4 } & & From & To & & 15,000 \\
\hline 9/15 & Stringing & $5484+00$ & $5636+00$ & 15,000 & 15,983 \\
\hline 9/16 & Stringing & $5636+00$ & $5705+83$ & 6,983 & 6,983 \\
\hline 9/17 & Stringing & $5705+83$ & $5806+00$ & 10,017 & 10,017 \\
\hline 9/18 & Stringing & $5806+00$ & $5972+00$ & 16,600 & 16,600 \\
\hline 9/19 & Stringing & $5972+00$ & $6140+00$ & 16,800 & 16,800 \\
\hline
\end{tabular}

Traditionally, the contractor analyzes productivities on a weekly basis for look-head scheduling. As mentioned above, the productivity of the future work is estimated as the average of the 3 to 5 proceeding weeks' productivity rates. In the proposed SLSM method, it is suggested that daily productivity rates be used in lieu of weekly rates in order to better reflect the variability of project performance. The following section discusses how to model this variability based on the collected daily productivity data

\subsection{Input Modeling}

In order to accurately represent the variability of an activity's performance, the productivity rate of an activity can be modeled as a probabilistic distribution ${ }^{4}$. The process of determining the underlying statistical distribution of an activity's productivity rate is an example of simulation input modeling, which provides a way for a scheduler to evaluate the variability in activity performance. To establish a statistical distribution to represent an activity's productivity, the abovementioned daily productivity data was used. The input modeling procedure involves two steps: distribution fitting and a goodness-of-fit test. The burden of the input modeling procedure can be greatly reduced by using commercially available input-modeling software. For this project, BestFit ${ }^{\circledR 9}$, recently incorporated into a risk-analysis program called @RISK, was used. For the purposes of this research, the program will be referred to hereinafter as BestFit.

\subsubsection{Distribution fitting}

The first step in modeling an activity's productivity rate is to generate a histogram to provide understanding of the characteristics related to type and shape of possible underlying distributions. Theoretical standard distributions, such as normal, beta, and triangular distributions, can be used to "test-fit" the data set and the histogram. Parameters of these distributions can be determined through this fitting process. Because of the use of computerized input-modeling software, a large number of standard distributions can be automatically fitted to the data set in a short period of time. For example, BestFit provides 28 different probability distributions for fitting a data set. These candidate distributions are ranked by the quality of fit, as described below.

\subsubsection{Goodness-of-fit test}

While any of the standard distributions can be used to represent the raw data, they can be very different in terms of how closely they represent the true underlying distribution of the productivity data. To test the quality of fit, there are heuristic procedures based primarily on visual inspection as well as goodness-of-fit methods based on statistical hypothesis tests ${ }^{7}$. To make input modeling easier to learn and use by industry practitioners, the goodness-of-fit test approach was adopted, because these tests are typically automated in input-modeling software. A goodness-of-fit test is a statistical hypothesis test that is used to determine if the raw data points are an independent sample from a particular statistical distribution. It describes statistically how well a standard distribution fits a set of raw data. Frequently used tests include the Chi-square test and the Kolmogorov-Smirnov test ${ }^{9}$.

While determining distribution parameters and selecting the best-fitted distribution is difficult to perform manually, input-modeling software such as BestFit automates this process and makes input modeling much more accessible to practitioners who lack knowledge in statistics. The input to BestFit is the daily productivity rate of an activity, as discussed previously. The output from BestFit is a list of ranked and parameterized distributions. A user can then pick the best-fitted distribution to represent the activity's performance. Table 3 shows the selected distributions along with their parameters for a group of repetitive 
activities. It should be noted that the data were scaled uniformly for confidentiality reasons.

Table 3. Selected input models.

\begin{tabular}{|c|c|}
\hline Task Name & Statistical Distributions \\
\hline Surveying & Exponential with mean $=16629$ \\
\hline Clearing & Exponential with mean $=9527$ \\
\hline Grading & $\begin{array}{l}\text { Normal with mean = } 2874 \text { and standard } \\
\text { deviation }=1363\end{array}$ \\
\hline Trenching & $\begin{array}{l}\text { Triangular with low limit }=670 \text {, most } \\
\text { likely }=1809 \text {, and high limit }=10720\end{array}$ \\
\hline Stringing & $\begin{array}{l}\text { Normal with mean }=4837 \text { and standard } \\
\text { deviation }=3011\end{array}$ \\
\hline Bending & $\begin{array}{l}\text { Beta with } a=2.3, b=3.4, \text { low }=670 \text {, and } \\
\text { high }=13812\end{array}$ \\
\hline Welding & $\begin{array}{l}\text { Beta with } \mathrm{a}=1.2, \mathrm{~b}=1 \text {, low }=700 \text {, and } \\
\text { high }=9800\end{array}$ \\
\hline Lower-in & $\begin{array}{l}\text { Normal with mean }=5882 \text { and standard } \\
\text { deviation }=3033\end{array}$ \\
\hline Tie-in & Exponential with mean $=2007$ \\
\hline Backfill & $\begin{array}{l}\text { Beta with a = 1.2, b = 2.9, low }=804 \text {, and } \\
\text { high }=15758\end{array}$ \\
\hline Clean up & $\begin{array}{l}\text { Normal with mean }=3688 \text { and standard } \\
\text { deviation }=1221\end{array}$ \\
\hline
\end{tabular}

\subsection{Simulation Modeling}

The pipeline construction operation can be modeled using the developed SLSM program. A Project element must first be defined. A user determines the default total work quantity in footage and the location of the external database for exporting simulation output. Within this Project element, a user can add multiple Activity elements to represent individual activities. These Activity elements can be linked together to show the precedence relationships between them.

For each Activity element, four user input parameters must be defined: planned start time, time buffer, space buffer, and productivity rate (e.g. ft/d). An activity's planned start time is measured as the number of days between the first day of the project and the first day of the planned start of an activity. Time buffer and space buffer are measured by days and space (e.g. footage), respectively, and are optional. The productivity rate can be specified as either a constant value or a statistical distribution, as discussed in the previous section.

To demonstrate the use of the SLSM program, two models were implemented: one model for the deterministic baseline schedule and the other for a simulated look-ahead schedule using SLSM. For the baseline model, activities' planned start times and productivity rates were quoted from the contractor's baseline schedule. For the look-ahead model, actual data and the distributions defined in Table 3 were used to represent the productivity rates. A screenshot of a sample SLSM model and a data-entry form for an Activity element are shown in Figure 3.
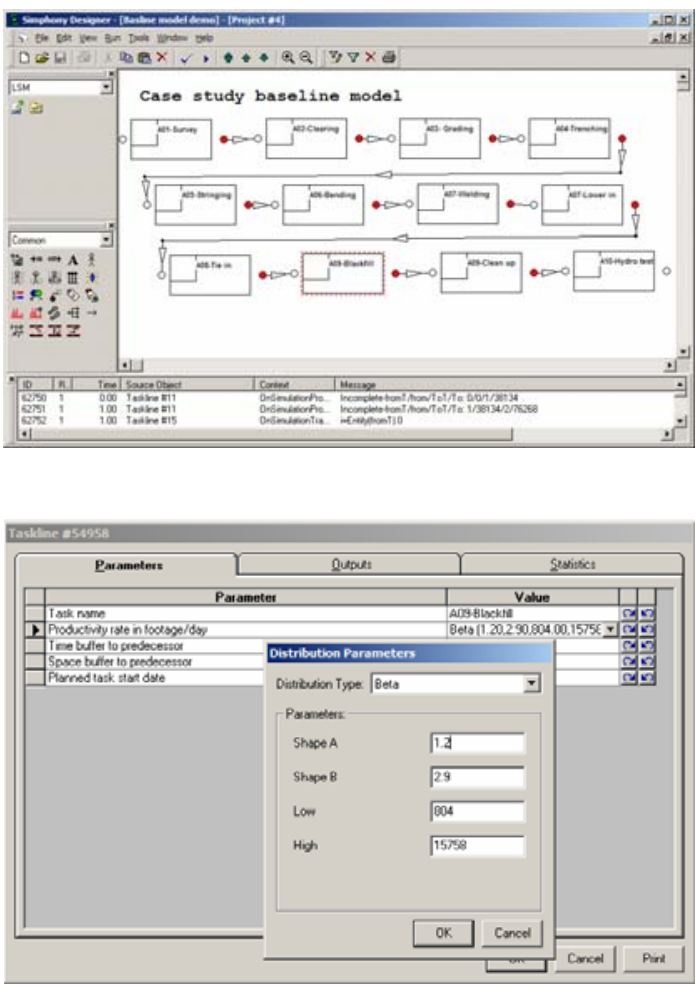

Fig. 3. An SLSM model and model inputs.

\subsection{Output Analysis}

After a model and its parameters are set, users can experiment with the simulation model and collect outputs. A simulation experiment usually contains a large number of runs, often as many as more than 30 . Each simulation run represents one observation of the possible future project performance and it predicts the project performance based on a set of random productivity rate values generated according to the distributions defined in Table 3 . The results from these simulation runs can then be gathered and used to forecast project as well as individual activity performance. This section demonstrates some sample outputs from simulation experiments.

Figure 4 shows a comparison of the deterministic baseline schedule (solid lines) and a simulated lookahead schedule (dotted lines). It can be seen that the actual productivity rates deviate from the deterministic estimates in the baseline schedule. Crew waiting or idle time are represented as horizontal line sections, which can be easily observed. The look-ahead schedule can be improved in different ways. For example, trench and tiein activity appears to be slow, and therefore, a more 
efficient configuration of the crew and equipment should be used. In addition, the planned start of some activities (e.g. hydro test) is too early, which will result in significant waste of crew idling due to conflict with the predecessors.

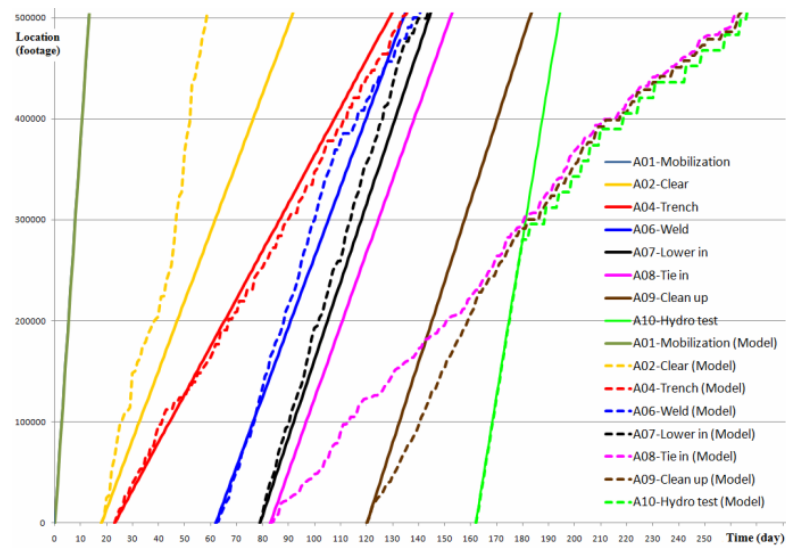

Fig. 4. A comparison of baseline schedule and simulated lookahead schedule.

Outputs from the simulated look-ahead schedule model can also be used to analyze the uncertainty of the total project duration. Each of the many simulation runs predicts a possible total project duration scenario. The duration data can be used to determine either the probability of completing a project within a certain number of days or the project duration corresponding to a certain desired risk level. Figure 5 shows the probability distribution of the total project duration. For example, according to this distribution, the probability of completing the project within 300 days is only $26 \%$, but the project has a $90 \%$ chance to complete within 334 days.

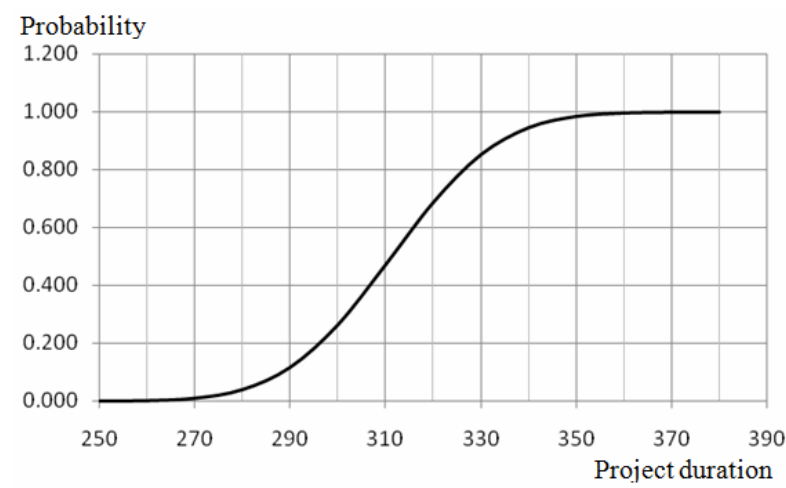

Fig.5. Probability distribution of total project duration.

Many observations related to an individual activity's productivity rate and idle time can also be obtained. As an example, the performance range chart can forecast the performance of an activity on a certain confidential interval. Figure 6 shows the performance range of the clean-up activity.

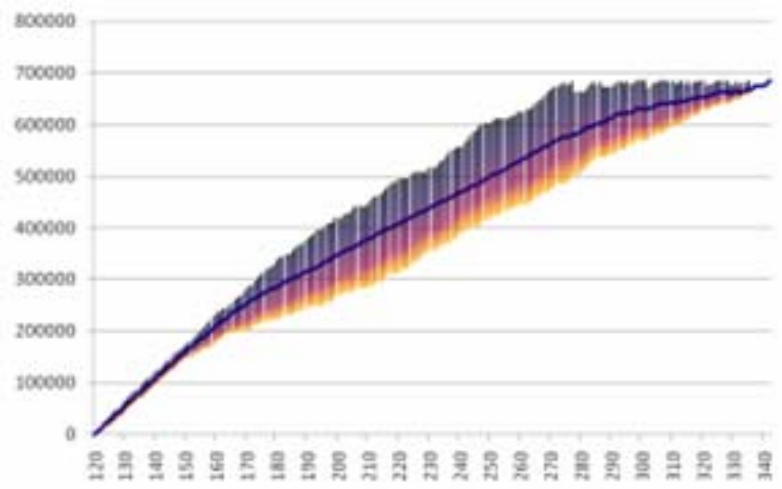

Fig. 6. Activity performance range chart.

\section{Conclusions}

Accurate and timely look-ahead scheduling based on the most up-to-date project data can greatly improve project execution and control. The traditional use of LSM is primarily limited to early project planning or master scheduling. After field operations commence, the most recent project performance data should be collected and analyzed for timely updating of the look-ahead schedule for more accurate forecasting that reflects the dynamic project environment. This research introduces LSM integrated with real-world performance data and computer simulation as an alternative tool for lookahead scheduling in linear construction projects. In the proposed method, actual performance data is first collected from ongoing construction operations and used to model the variability of activity performance. This variability is then incorporated into a process simulation model that can be used to generate lookahead schedules. A computer program, SLSM, was designed and implemented. SLSM allows schedulers to model and analyze repetitive linear construction projects while considering variability in activity performance. Various data analyses can be performed based on the simulation output data-e.g., evaluating baseline schedule, the total project duration, individual activity performance, and idle time. The case study demonstrates that the proposed approach can effectively utilize real-world data to model and forecast the impact of uncertainty on future performance. Furthermore, better capability in forecasting future project performance based on actual productivity data can help a scheduler anticipate problem areas and formulate new plans to improve project performance. 


\section{References}

1. J.W. Hinze, Construction Planning and Scheduling, 3rd ed., (Pearson Prentice Hall, Upper Saddle River, NJ, 2008).

2. R.A. Yamin and D. J. Harmelink, Comparison of linear scheduling method (LSM) and critical path method (CPM). Journal of Construction Engineering and Management, 127(5) (2001), pp. 374-381.

3. C. Srisuwanrat and P. G. Ioannou, The investigation of lead-time buffering under uncertainty using simulation and cost optimization”, Proc. IGLC-15 (Ann Arbor, MI, 2007).

4. W. Liu, I. Flood, R. A. Issa, Simulation and optimization of linear construction projects. Proc. of Computing in Civil Engineering, ASCE (Cancun, Mexico, 2005).

5. G. A. Duffy, G. D. Oberlender, D. Jeong, Linear scheduling model with varying production rates, Journal of Construction Engineering and Management, 137(8), (2011), pp. 574-582.

6. S. M. El-Sayegh, Linear construction planning model (LCPM): A new model for planning and scheduling linear construction projects. Ph.D. dissertation, Texas A\&M Univ., (College Station, TX, 1998).

7. A.M. Law and W. D. Kelton, Simulation Modeling and Analysis, 3rd ed., (McGraw-Hill, New York, 2000).

8. D. Hajjar and S.M. AbouRizk, Unified modeling methodology for construction simulation, Journal of Construction Engineering and Management, 128(2) (2002), pp. 174-185.

9. Palisade Corporation, BestFit User Manual, (Palisade Corporation, Ithaca, NY, 2004). 